Conclusions Routine prenatal syphilis screening has identified 14/48 women who required PCN treatment, all of whom received PCN prior to delivery with only 1 woman experiencing a possible adverse event. The only congenital case occurred in a mother with no prenatal care, suggesting a need for a strategy to identify marginalised women with syphilis early in pregnancy. Although the average time to contact these patients was short, the time to administration of 1 st dose of PCN was longer, reflecting the need to educate women about the importance of prompt and complete therapy in preventing congenital syphilis.

\section{P1-S6.10 ACCEPTABILITY OF ANAL PAP SELF-SCREENING IN HIGH-RISK WOMEN: FINDINGS FROM ENGLISH AND SPANISH FOCUS GROUPS IN NORTHERN CALIFORNIA}

doi:10.1136/sextrans-2011-050108.234

${ }^{1} \mathrm{C}$ McNeil, ${ }^{2} \mathrm{C}$ Piñera, ${ }^{1} Y$ Maldonado, ${ }^{3} \mathrm{~V}$ Levy. ${ }^{1}$ Stanford University, Stanford, USA; ${ }^{2}$ Hospital González Cortez, Universidad de Chile, Santiago, Chile; ${ }^{3}$ San Mateo County Health System, San Mateo, USA

Background HPV has a causative role in anogenital malignancies. During 2003-2007, the rate of anal cancer for women of all races in California was 2.2/100000 compared to a national rate of 1.8/ 100000 . There are no national screening measures for preventing anal cancer, a rare disease that affects more women than men annually. Screening approaches have mainly been studied in men. In preparing for an anal cancer self-screening pilot study in high-risk women, we conducted focus groups in English and Spanish to assess HPV knowledge and acceptability and comprehension of anal Pap instruction materials. Qualitative data regarding acceptability and feasibility of anal pap screening in women have not previously been reported.

Methods Women who were biological females $>18$ years of age and fluent and literate in English or Spanish were recruited from a public HIV or STD clinic for participation in focus groups conducted by two English and Spanish speaking moderators. Participants were asked a structured list of open ended questions on HPV knowledge, and the acceptability of study forms including an illustrated anal PAP instruction sheet. Qualitative data was collected and reviewed for common themes and emphasis.

Results Two focus groups included 6 English speaking (ES) women and 8 Spanish speaking (SS) women. Knowledge gaps identified for SS women included: basic anatomical terms, HPV can infect both women and men, HPV is a STI, HPV can cause cancer, and the existence of a preventive HPV vaccine. Stigma was identified as an issue associated with STI education for SS women only. Shared knowledge gaps for ES and SS women included: asymptomatic nature of HPV, symptoms potentially caused by HPV, and that warts can turn into cancer. Both groups agreed public health HPV campaigns should target both men and women. Whereas ES women encouraged a more media based approach to HPV education, SS women commented current campaigns are too vague and emphasised a more personal, interactive approach to HPV education in public venues. Self-sampling was viewed positively by participants, along with self-sampling instructions; some modifications to collection materials were suggested.

Conclusions Focus groups revealed significant knowledge gaps in HPV associated malignancies and cancer screening in high-risk females. Anal PAP self-screening appears to be an acceptable approach; however, the large scale implementation of such strategies may require targeted educational campaigns particularly in underserved communities.

\section{P1-S6.11 PREVENTION OF MOTHER TO CHILD TRANSMISSION OF HIV (PMTCT) PROGRAM IN BURKINA FASO: HOW HIGH IS THE COVERAGE OF VOLUNTARY COUNSELLING AND HIV TESTING (VCT) SERVICES WITHIN A CLINICAL TRIAL SUPPORTING ENVIRONMENT?}

doi:10.1136/sextrans-2011-050108.235

${ }^{1} \mathrm{H}$ A Traore, ${ }^{1} \mathrm{E}$ Some, ${ }^{1} \mathrm{~N}$ Meda, ${ }^{2} \mathrm{~N}$ Nagot, ${ }^{2} \mathrm{P}$ Van De Perre. ${ }^{1}$ ANRS/Burkina Faso, Ouagadougou, Burkina Faso; ${ }^{2}$ INSERM U 1058/France

Background Since 2003, Burkina Faso has set up a national PMTCT program. Programme monitoring 2009 annual report showed high health districts and facilities coverage. But at beneficiaries' level, how many women and children in need of PMTCT interventions have really access? The objective of our study was to measure the uptake of VCT with a comprehensive set of data collected in the recruitment process in a clinical trial evaluating postnatal chemoprophylaxis to reduce MTCT.

Methods We carried out a cross-sectional study from 1 January 2010to 31 December 2010 in 26 out of 35 PMTCT sites in five health districts in Ouagadougou city. Weekly data collection in PMTCT registers and semi-structured interviews with the personals in charge of $\mathrm{MCH}$ departments.

Results Among the 44484 new recorded Antenatal care (ANC) attendees, 37539 received HIV counselling and 37489 were tested for HIV (results returned immediately), an acceptance rate of 99, $86 \%$. 6,945 new ANC did not profit from the HIV counselling equalling $15.61 \%$ of the participant population. This miss opportunity for VCT was related to test supplies out of stock (78\%), lack of VCT offer due to opt in strategy still in place in many facilities $(17 \%)$ and structural problems (no trained staff, lack of infrastructures) in the remaining cases.

Conclusion Our results underline the overall good performance of the PMTCT program in the context of a clinical trial facilitating environment. However, a better organization of the supply procurement would allow improving VCT coverage rate. With this high coverage of VCT in research context, we can assume that the low VCT coverage at program (75\%) level is mainly due to healthcare system problem.

\section{P1-S6.12 THE CONTRIBUTION OF A CHLAMYDIA SCREENING PROGRAMIME TO TESTING AND CASE-FINDING IN ADDITION TO REGULAR STI-CARE IN THREE REGIONS OF THE NETHERLANDS}

doi:10.1136/sextrans-2011-050108.236

${ }^{1}$ I V F van den Broek, ${ }^{2} \mathrm{H}$ M Götz, ${ }^{3} \mathrm{E}$ E H G Brouwers, ${ }^{4} \mathrm{H}$ S A Fennema ${ }^{3} \mathrm{C} J$ P A Hoebe, ${ }^{4} \mathrm{R}$ H Koekenbier, ${ }^{5} \mathrm{~L}$ L Pars, ${ }^{2} \mathrm{~S} M$ van Raveseijn, ${ }^{6} \mathrm{E}$ L M Op de Coul, ${ }^{5} \mathrm{~J}$ E A M van Bergen. ${ }^{1}$ Centre for Infectious Disease Control, National Institute of Public Health and the Environment, Bilthoven, Netherlands; ${ }^{2}$ Division of Infectious Disease Control, Rotterdam Rijnmond Public Health Service, Rotterdam, Netherlands, ${ }^{3}$ Department of Infectious Diseases, South Limburg Public Health Service, Geleen, Netherlands; ${ }^{4}$ Cluster of Infectious Diseases, Department of Research, Online Research and Prevention Unit, Amsterdam Health Service, Amsterdam, Netherlands, ${ }^{5}$ STI AIDS Netherlands, Amsterdam, Netherlands; ${ }^{6}$ Epidemiology \& Surveillance Unit, Centre for Infectious Disease Control, National Institute of Public Health and the Environment, Bilthoven, Netherlands

Background The impact of a Chlamydia screening programme can be measured by the incremental amount of Chlamydia tests performed and cases detected as compared to the throughput in regular care. In the Netherlands, regular STI care is provided by specialised STI-centres (aimed at high-risk groups) and General Practitioners (GP's, basic opportunistic screening and care for 